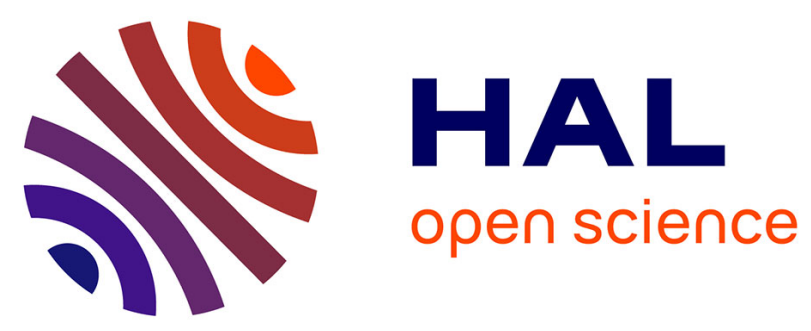

\title{
Les colonies latines de Narbonnaise : un nouveau document d'Arles mentionnant la Colonia Iulia Augusta Avennio
}

\author{
Michel Christol, Marc Heijmans
}

\section{To cite this version:}

Michel Christol, Marc Heijmans. Les colonies latines de Narbonnaise: un nouveau document d'Arles mentionnant la Colonia Iulia Augusta Avennio. Gallia - Fouilles et monuments archéologiques en France métropolitaine, 1992, 49, pp.37-44. 10.3406/galia.1992.2927 . hal-01915205

\author{
HAL Id: hal-01915205 \\ https://hal.science/hal-01915205
}

Submitted on 19 Jan 2020

HAL is a multi-disciplinary open access archive for the deposit and dissemination of scientific research documents, whether they are published or not. The documents may come from teaching and research institutions in France or abroad, or from public or private research centers.
L'archive ouverte pluridisciplinaire HAL, est destinée au dépôt et à la diffusion de documents scientifiques de niveau recherche, publiés ou non, émanant des établissements d'enseignement et de recherche français ou étrangers, des laboratoires publics ou privés.

\section{(1) (1) $\$$}

Distributed under a Creative Commons Attribution - NonCommercial - NoDerivatives 44.0 


\title{
Les colonies latines de Narbonnaise : un nouveau document d'Arles mentionnant la Colonia Iulia Augusta Avennio
}

\author{
par Michel CHRISTOL et Marc HEIJMANS
}

\%a découverte, en Arles, d'une inscription mentionnant la colonia Iulia Augusta Avennio permet d'établir que cette colonie latine fut fondée à l'époque augustéenne, avant de devenir colonie romaine sous Hadrien, pui que l'authenticité de CIL XII, 1120 (Apt) semble à présent établie. Dans la liste de Pline (NH, III, 36) elle est incluse comme colonie latine dans l'énumération des oppida latina. C'est l'occasion d'examiner la composition des listes fournies par cet auteur, en ordre chronologique pour les colonies romaines, en ordre alphabétique pour les colonies latines, conformément à la source utilisée, et d'étudier les diverses titulatures épigraphiques des colonies latines : on peut distinguer les colonies Iuliae, antérieures à 27 avant J.-C. et les colonies Iuliae Augustae, postérieures à cette date, et les insérer dans un mouvement de création qui s'étend en plusieurs étapes depuis l'époque césarienne.

Thanks to the find in Arles of an inscription mentioning the colonia Iulia Augusta Avennio, we can establish that a colony was founded there in the Augustean age, before becoming a roman colony under Hadrian, since the authenticity of CIL, XII, 1120 (Apt) is now beyond doubts. In Plinius' list $(\mathrm{NH}, I I I, 36)$ it is included as a latin colony among the oppida latina. This gives an opportunity to scrutinize the control of the lists gathered by this author, most of which are ordered chronologically for the roman colonies, alphabetically for the latin colonies, according to the sources, and to study the different epigraphical titulatures of the latin colonies : it is possible to distinguish between the Iuliae colonies, dating from before 27 B.C., and the Augustae colonies, dating after this date, and to give them their place in a long cycle of creations which covers several stages from the times of Caesar.

Mots clés : Narbonnaise, colonies romaines, colonies latines, statut municipal d'Avignon, Pline le Naturaliste. 
Plusieurs inscriptions ont èté découvertes dans la ville d'Arles durant les fouilles de la nécropole du cirque romain, appelée aussi nécropole du Plan-duBourg (campagne 1989), sur l'emplacement où šélève à présent l'Institut de recherches sur la Provence antique (IRP:I) ${ }^{1}$. Mais l'extrème pillage du site archéologique explique l'état fragmentaire des documents, souvent réduits à quelques lettres ${ }^{2}$. Quelques-uns cependant sont moins incomplets que les autres. Deux d'entre eux, précieux pour l'histoire sociale de la colonie, seront publiés ailleurs ${ }^{3}$. Un troisième présente un intèrêt qui dépasse le cadre local.

L'inscription a été reconstituée en raccordant deux fragments mis au jour dans un niveau d'abandon, voire de pillage, que l'on peut dater du $v^{e} s$. après J.C. ${ }^{4}$. Il s'agit de la partie inférieure gauche d'une plaque de marbre de bonnes dimensions si l'on considère que le texte gravé à gauche devait être accompagné, à sa droite, d'un second texte inséré dans un champ épigraphique de dimensions équivalentes.

Inventaire : IRP 89. 22:211.612.

Dimensions (en $\mathrm{cm})$ longueur : 59; hauteur : 18.5 environ; épaisseur: 1,5. Champ épigraphique conservé (a gauche) longueur : 42 ; hauteur : 10.5) environ. Hauteur des lettres -... ligne 1 (conservée) : 2.6 a 3.5): ligne $2: 2,4$. On peut estimer la largeur totale de la plaque de marbre a $108 \mathrm{~cm}$ environ.

Bibliographie: M. Heismaxs, Epigraphie et matériel lapidaire, in : (. Sintìs (éd.), Carnets de fouilles d'une presqu'ile, Renue d'Arles, 2, 1990, n" 1 et fig.. p. $: 20-21$.

1 Ces fouilles ont commencé dès 1984 sous la direction de C. Sintès. Sur les découvertes et la progression des chantiers, voir les rapports dans Gallia. 44, 1986, p. 394-397; Gallia Informalions, 1987-1988. 2, p. 2:34-236. Sur la nécropole. M. Euzhnnat, (i. Malofer, la nécropole du cirque, in: C. sintiss (éd.), Du noumeau sur l'Arles antique, Revue d'Arles, 1 , 1987. p. 11.1-117. Sur les premiers résultats des fouilles récentes, voir C. Sintiss, Le résultat des fouilles, in : C. Sinrìs. (éd.). Carnets de fouilles d'une presqu'ile, Revue d'Arles, 2, 1990. p. 11-15.

2 Il existe en tout 21 documents latins et 4 documents grecs. Le catalogue sera fourni dans la publication définitive des fouilles.

3 Première publication par M. His.s.sans, Épigraphie ef matériel lapidaire, in : C. Sintis (éd.). Carnets de fouille d'une presquile. Revue d'Arles, 2, 1990, p. 21-22 (n" 2), p. 22 $\left(n^{\prime \prime} 3\right)$.

4 On corrigera. sur ce point. les indications fournies dans la premiere publication. Vérification faite. ce niveau doit ètre mis en relation aver l'installation de l'habitat tardif dans les alvéoles du cirque : nous sommes alors au v"s. Le peu de materiel trouvé en contact direct avec ces fragments (sigillée (laire I)) confirme cette datation.

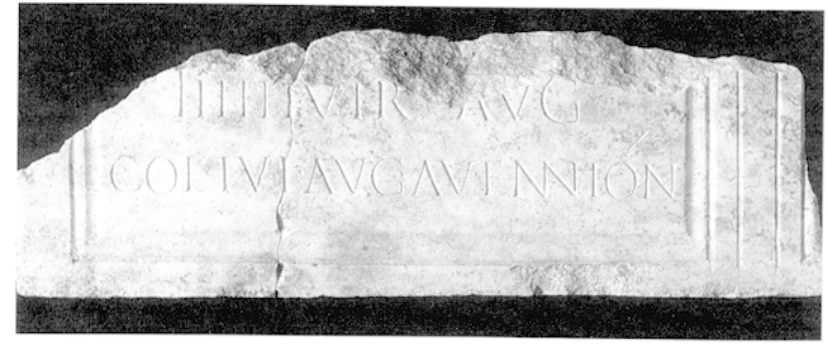

(cliché M. Lacanaud, Musée Réattu).

(se)vir(o ou -i) Aug(ustali ou -is)
col(onia) Iul(ia) Aug(usta) Avennion(e)

Mise en page soignée. Très belle écriture, avec effets de dissymétrie. Les mots sont séparés par de petits points triangulaires. A la ligne 1 conservée, un tiret allongé devait surmonter les barres médianes du chiffre six. A la dernière ligne, ligature des deux $\mathrm{N}$ d'Aennion(e), apex sur le $O$.

L'existence d'un double champ épigraphique s'accorde peu avec l'appartenance à un monument de caractère public ou honorifique. Il s'agit plutôt d'un document funéraire, constitué de deux épitaphes juxtaposées. Les mots de la dernière ligne complètent donc ceux de la ligne précédente, mais on ne sait s'il faut utiliser le datif ou le génitif (celui-ci, en particulier, dans l'hypothèse d'une mention des mànes). Le défunt avait été sévir augustal dans la cité d'Avignon quoiqu'il soit vraisemblablement arlésien, ou tout au moins résidant dans cette colonie, puisque son tombeau avait été élevé aux abords de la ville qui était le chef-lieu des Sextani. Quant à la date, celle du $\mathrm{I}^{\mathrm{er}} \mathrm{s}$. après $\mathrm{J} .-\mathrm{C}$. pourrait convenir : comme on le verra plus bas, on ne peut dépasser le premier quart du II" s. après J.-C.

L'intérèt de ce document est double. D'abord on y trouve la deuxième mention d'un sévir augustal avignonnais, malheureusement anonyme, ce qui interdit toute etude sociale 5 . Peut-être, toutefois, avait-il obtenu également le sévirat augustal dans la cité d'Arles, à l'instar d'autres personnages qui revêtus de cet honneur en cette colonie l'avaient aussi reçu ailleurs, tels P. Sextius Florus (à Aix et Arles), Aebutius Agathon (a Arles et $A p t$ ), et M. Silenius Symphorus (a Lyon, Arles et Riez) ${ }^{6}$. Surtout l'ins-

¿ Lautre sevir augustal avignonnais, C. Pontinius [---]io. est mentionné dans CII XII, 5825.

(i) CIL XII. 705: CIL XII. 1005: ILT( 24l. Sur les deux premiers personnages, L.-A. Cosstans, Arles antique, Paris, 1921, p. 86-88. 
cription transmet pour la première fois, à une date assez haute, la titulature de la colonie d'Avignon au cours d'une phase précoce de son histoire institutionnelle : colonia Iulia Augusta Avennio?.

Jusqu ici, pour comprendre quelle avait été l'évolution juridique de cette cité, on en était réduit aux commentaires de deux textes et d'une inscription. Le texte dont la date est la plus ancienne se trouve dans l'Histoire naturelle de Pline l'Ancien, adressée à Titus en 77 après J.-C. ${ }^{8}$. Là se trouve la mention d'Avennio Cavarum au sein de la liste des oppida latina que l'auteur place in medilerraneo dans la province de Gaule Narbonnaise ${ }^{9}$. Mais on a estimé habituellement que Pline ne décrivait pas la situation de son époque et qu'il reprenait pour l'essentiel les Commentaires d'Agrippa ${ }^{10} \mathrm{ou}$, mieux, des listes etablies durant le principat d'Auguste ${ }^{11}$. Cet auteur nous livrerait donc la première étape de l'histoire administrative d'Avignon. I.c second texte, plus récent, se trouve dans la Géographie de Ptolémée ${ }^{12}$, composée dans le deuxième quart du II"s. après J.-C., plutôt vers 130: il mentionne Aoúevvíwv xo $\omega \omega^{\prime} x^{13}$. Quant à l'inscription, il s'agit d'un docu-

7 On ne peut restituer qu'Auennion(e), ablatif de lieu. sous la forme d'un adjectif ou d'un adjectif substantivé, lethnique est Avenniensis (CIL XII, 3169: Avennien/ses patrono?]: CIL. XII. 3275 : curatori Cabel/lens./, Avenniens.. Foroiuliens.. Aptenses patrono: cf. (.II, XII, p. 130). II n’apparait pas ici. La titulature d'Avignon revèt donc la forme normale par laquelle, après la mention d'une magistrature ou d'un honneur, est dénommée une cité, celle de l'ablatif de lieu ou du locat if de la ville qui en est le chef-lieu : CIL XII, 705 ((se)vir. Aug. col. Jul. Aquis): 982 ((se)vir. Aug. col. Iulia Aquis Sex(is) : 1005 ((se)viro col. Iul. Aptae) ; 3200 (decurioni ornamentario col. Aug. Nemausi): 32033 (ornamentis dec. honoratus col. Aug. Nemausi. (se)vir Aug. col. Copia Claud. Lugdun. item Varbone Martio et Firm. Iul. Secund. Arausione et Foro Iulii Pacalo); 4:363 (aed. Aquis Iulis), etc. Lorsqu'un génitif pluriel apparait, c'est que le nom du peuple est incorporé dans le nom de la colonie : CIIL XII, 982 ((qualtuor)vir., pontif. col. Reior. Apollinar(e)): 1581 ((se)viri / Augustalis Dea/ Aug. Vocontiorum) : 3200 (decurioni col. Apollinare Reiorum) ; 3290 ((se)vir. Aug. corporat. Dea Aug. Vocontior.); 3291 (decurion. col. Iul. Aug. Apollinar(e) Reior.).

8 P.-M. I)vval, La Gaule jusqu'au milieu du ve siècle, Paris, 1971 (Les sources de l'histoire de France des origines à la fin du $x v^{\circ}$ siècle, I), p. 368-379.

9 PI.Ife. VII. III, 36:- G. BarRuol., Les peuples préromains du Sud-Est de la Gaule. Etude de géographie historique, Paris, 1975, 1'r suppl. à la Revue archéologique de Narbonnaise. p. 16-2. 4 .

10 Sur ces documents, en dernier lieu voir : C.. Nicolet, Linventaire du monde. Géographie et politique aux origines de lempire romain. Paris, 1988. p. 112-113.

11 C. Nicolet. op. cit. p. 181-192.

12 O. Strix, Zur Datierung von Ptolemaios Geographie, Philologus. 81, 1925, p. 117-125.

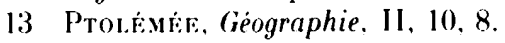

ment mis au jour dans la cité d'Apt ${ }^{14}$, mais dont l'authenticité a été mise en doute par Hirschfeld, puis plus récemment par M. Zarhnt ${ }^{15}$. Aussi le premier de ces savants demeurait-il dans l'incertitude quant à l'évolution administrative de la cité ${ }^{16}$. En revanche, Fr. Vittinghof qui mettait aussi en valeur, dans l'œuvre de César. la diffusion du droit latin en Narbonnaise, considérait pour sa part qu'Avignon, cité latine d'après Pline, n'aurait pas nécessairement perdu le droit latin en devenant colonie, comme l'indique Ptolémée ${ }^{17}$.

D'autre part, ceux qui accordent crédit à l'inscription d'Apt déduisent du titre colonia Iulia Hadriana Avennio et de la présence de duumvirs que la cité fut à un certain moment de son histoire une colonie romaine. Toutefois les avis divergent sur les modalités de l'évolution et sur la chronologie de celle-ci. C. Jullian, par exemple, considérait que la colonic romaine d'Avignon avait été implantée lors d'une deuxième phase de la colonisation de droit romain, sous Auguste: il la datait de 16-15 avant J.-C. ${ }^{18}$. Elle serait donc venue bien après la vague césarienne ${ }^{19}$, et, dans son esprit, elle aurait même été postérieure à la rédaction des Commentaires d'Agrippa, dont il faisait la source de Pline ${ }^{20}$ : de la

14 CIL. XII, 1120: I)(is) m(anibus) L(ucio) Volus(io) L(uci) f(ilio) Severiano. (quattuor)vir(o) clolonia) I(ulia) Apt(a) (bis) el flam(ini) item (duo)vir(o) c(olonia) I(ulia) Madr(iana) Avenn(ione) et pontif(ici), sacerdot(i) Vrbis Rom(ae) aetern(ae), Vol(usia) Severiana patri incomparabili.

15 CIL. XII, p. 130 et commentaire ad 1120 . C. p. 932 (index) ou tous les renseignements fournis par le texte sont affectés d'un astérisque. Toutefois, quelques années après, le texte fit l'objet d'une tentative de pleine réhabilitation de la part de Pall.u DE Lessent, Bullelin de la Sociëlé Nationale des Antiquaires de France, 1916, p. 283-287. Mais récemment le principe du faux a été remis en honneur par M. Zahrvt, Vermeintliche Kolonien der Kaisers Hadrian, Zeilschrift für Papyrologie und Epigraphik, 71, 1988, p. 229-249, part. p. 228-233, en sappuyant sur les modes de dénomination des cités.

16 CII. XII. p. 130 : dans l'intitulé du chapitre introductif sur Avignon, Hirschfeld se contente du mot Avennio. Il écrit dans la notice qui suit : "inter oppida Latina recensetur a Plinio (l.c.); coloniam eam appellat Ptolemaeus, cum in titulis excepto uno Aptensi eoque fraudis suspicioni valde obnoxio Ilvir(i) c(oloniae) I(uliae) Hadr(ianae) Avenn(iensis) (infra n. 11:0) nec municipii nec coloniae nomine exornetur".

17 Fr. Virringhof, Römische Kolonisation und Bürgerrechtspolitik unter Caesar und Augustus (Akad. der Wissenschaften und der Literatur, Abhandl. der Geistes- und Sozialwissenschaften Kl.), 14. 1951, p. 1280-1282, avec surtout note 1 p. 1281. Sur le droit latin octroyé par César. voir déjà $R E$. ? (1896) col. 2281. s.v. Avennio (Iнм).

18 C. Jullian, Histoire de la Gaule, IV, Paris, 1921. 2réd.. p. 77 et note 1, p. 78 et note 4 .

19 C. Jullian, op. cit.. IV, p. 30-35.

20 Abordant la question du statut de ces nouvelles fon- 
sorte on aurait assisté très tôt à la substitution d'une colonie romaine à l'oppidum latinum initialement organisé, mais dont le souvenir avait été maintenu par le Naturaliste parce qu'il avait repris une source composée à date trop haute pour enregistrer la mutation de statut dont nous venons de parler. A la vérité, comme le reconnaissait lui-même cet historien, une telle reconstitution n'expliquait pas l'ajout du surnom Hadriana dans la dénomination de la colonie $^{21}$. Aussi d'autres auteurs, tout en mettant en valeur le schéma évolutif oppidum latinum-colonie latine-colonie romaine, déduisent que l'ultime élévation résulta d'une décision d'Hadrien, et que la phase intermédiaire s'ouvrit sous le principat de Claude ${ }^{22}$. Seul, récemment, A. L. F. Rivet a fait remarquer que si l'on acceptait l'authenticité de l'inscription d'Apt, il fallait attribuer la création de la colonie latine à l'époque augustéenne : "the title, if correct, surely points to Augustan times" ${ }^{23}$.

Sur ce point le cheminement de l'auteur est trop rapide et sa conclusion trop générale. Puisque l'on ne peut extraire de l'inscription d'Apt que le nom Iulia sans le surnom Augusta, on admettra que les «Augustan times" doivent être entendus dans le sens le plus large, de 43 avant J.-C. à 14 après J.-C., et qu'il ne faut point exclure une date antérieure à 27 avant J.-C. Mais l'on sait par ailleurs que durant cette longue période, l'histoire de la colonisation des provinces, y compris en Narbonnaise, fut complexe et put se dérouler en plusieurs phases. Aussi, tout en mettant en évidence les débuts du principat, A. L. F. Rivet ne pouvait apporter toutes les précisions souhaitées. Désormais, l'inscription d'Arles lève toute incertitude. Elle montre qu'une étape importante de l'histoire de la cité d'Avignon se plaça après l'année 27 avant J.-C., quand le prince reçut le surnom d'Auguste : on peut le déduire du nom de la

dations, C. Jullian estimait qu'elles s'ètaient produites dans le cadre du droit romain. Cette conclusion, faut-il remarquer, est à l'origine de bien des points de vue émis dans l'école française sur le contenu de la colonisation aux débuts de l'époque impériale. De plus, cet auteur (op. cit., p. 76-77) envisageait un système complexe qui aurait articulé le droit romain pour la ville et le droit latin pour le territoire, cf. p. 77 et note 1, p. 244246 .

21 C. Jullian, op. cit., IV, p. 471 et note 3 . Il est fidèlement suivi par J.-J. HATt, Hisloire de la Gaule romaine, Paris, 1959, p. 91-92, ainsi que par M. Labroussf, Toulouse anlique des origines à l'élablissement des Wisigoths, Paris, 1968, p. $488-493$.

22 S. Gagnière, J. Granier, Avignon de la préhisloire à la papauté, Avignon, 1970 , p. 86 , repris par R. Chevali.ier, Provincia, Paris, 1982, p. 83.

23 A. L. F. Rivet, Gallia Narbonensis. Southern Gaul in Roman Times, Londres, 1988, p. 265. colonia Iulia Augusta Avennio, dans lequel l'élément Iulia Augusla, à notre avis indissociable ${ }^{24}$, se réfère à une création proprement augustéenne et non triumvirale. C'est alors que la colonie d'Avignon s'ajouta au groupe des colonies latines déjà existantes.

On doit la retrouver dans la liste des oppida latina de Pline le Naturaliste. Certes cet auteur reprenait un document qui avait été mis au point pour la première fois à l'époque augustéenne ${ }^{25}$. Mais il était à jour pour les colonies de droit romain comme pour la liste des peuples provinciaux. Ne mentionne-t-il pas à ce sujet que sous Galba une modification de frontière du côté des Alpes maritimes avait fait passer en Narbonnaise les Avantici et les Bodiontici : adiecil formulae Galba imperator ex Inalpinis Avanticos atque Bodionticos, quorum oppidum Dinia ${ }^{26}$ ? Et que penser de la liste des colonies de droit romain qui enregistre toutes ces communautés, d'Arles à Vienne? La première place d'Arles n'étonne pas puisqu'elle est fondation césarienne, attestée par Suétone ${ }^{27}$. Mais Vienne (Vienna Allobrogum chez Pline), citée en dernier lieu, n'avait-elle pas été promue au rang de colonie de droit romain durant le règne de Caligula comme l'avaient supposé depuis longtemps H.-G. Pflaum et A. Chastagnol, relayés plus récemment par $R$. Frei-Stolba et A. L. F. Rivet ${ }^{28}$ ? Aussi renoncera-t-on à supposer

24 Il est plus difficile d'envisager une évolution comparable à celle de Vimes, colonie latine césarienne qui reçut plus tard le titre d'Augusta (Chr. Goudinfau, Le statut de Nimes et des Volques Arécomiques, Revue archéologique de Narbonnaise, 9,1974, p. 114). Cela n'indiquait nullement pour cette cité un changement de statut, mais un bienfait du prince, que l'on peut placer en 16-15 avant J.-C., lors de la construction de l'enceinte (M. Christor, Chr. Goudinfau, Nimes et les Volques Arécomiques au $\mathrm{I}^{\mathrm{er}} \mathrm{s}$. avant J.-C., Gallia, 45, 1987-1988, p. 97-99 et p. 102). Or jamais le nom Iulia n'apparaît dans la titulature de la cité (col. Nem. ou col. Aug. Nem.). Comme l'on sait qu'il y eut diverses phases de colonisation dès Cèsar, puis sous son successeur, d'abord comme triumvir puis comme Auguste (cf. infra note 54), il semble préférable d'ordonner les créations de colonies d'après l'évolution de la dénomination du princeps.

25 M. Christol, Chr. Gounineau, op. cit., p. 90 et p. 99. Voir aussi, sur les sources de Pline, C. Nicoler. L'origine des regiones Italiae augustéennes, Cahiers du Centre G. Glolz, 2, 1991, p. 85-90.

26 PI.INe, VII, III, 37.

27 Surtone, Tib., 4 ; - Chr. Goudineau, Note sur la fondation de Lyon, Gallia, 44, 1986, p. 171-173.

28 II.-G. PFiauM, La mise en place des procuratèles financières dans les provinces du llaut-Empire, Revue historique de droit français et étranger, 46,1968, p. $378 ;-$ А. СнаsTakinol, Les modes d'accès au sénat romain au début de l'Empire, Bulletin de la Sociète Vationale des Antiquaires de France, 1971, p. 291-292: - R. Frei-Stoi.ba, Zum Stadtrecht von Vienne (Colonia Iulia Augusla Florentia Vienna), Museum Hel- 
que la liste des colonies de droit romain (Pline, $\mathrm{NH}$, III, 36 : in mediterraneo coloniae Arelate Sextanorum, Baeterrae Septimanorum, Arausio Secundanorum, in agro Cavarum Valentia, Vienna Allobrogum) avait été bàtie sur le modèle de celle qui enregistrait les oppida latina, c'est-à-dire en ordre alphabétique, ce qui contraignait à admettre que Pline avait pu placer Béziers (Baeterrae) avant Orange (Arausio) en modifiant l'énumération qui se trouvait dans sa source ${ }^{29}$. Plutôt, fidèle à la réalité du moment, celle-ci avait été bâtie en ordre chronologique, c'està-dire qu'elle avait enregistré les fondations et les promotions au fur et à mesure qu'elles étaient survenues : elle révélait que la source de Pline, la formula provinciae qu'il citait ailleurs, présentait en ce chapitre les mêmes caractéristiques d'énumération. Celle-ci était donc le fruit d'une construction progressive ${ }^{30}$.

Faut-il alors récuser l'idée que la liste des oppida latina serait aussi à jour? Bâtie à un certain moment en ordre alphabétique (la digestio in litteras) ${ }^{31}$, n'offrirait-elle pas chez Pline un état du groupe des communautés de droit latin, dans toute sa diversité, à l'époque flavienne? Certes l'expression oppidum latinum est un vocable désormais archaïque à l'époque de Pline : mais ce caractère insolite rappelle le moment déjà ancien où ces structures furent mises en place ${ }^{32}$, comme il montre aussi qu'il n'était pas encore nécessaire de lui substituer un terme neuf. Ve peut-on donc pas admettre comme assuré que

veticum, 41, 1984, p. 81-85; - A. L. F. Rivet, op. cil., p. 306. Pace A. Peldetier, Vienne antique de la conquête romaine aux invasions alamanniques, Roanne, 1982, p. 73-80.

29 Tel est l'avis de O. Cuntz, De Augusio Plinii Geographicorum Auctore, Bonn, 1888, p. 13 avec note $2:$ "Baeterrae ante Arausionem a Plinio ipso fortasse sunt positae».

30 Aussi supposera-t-on que la fondation de Béziers prècède celle d'Orange, à l'époque triumvirale, l'une et l'autre pouvant se placer en 36 et 35 avant J.-C. respectivement, comme le voulait A. Piganiol, Les documents cadastraux de la colonie romaine d'Orange, Paris, èd. du C.NRS, 1962, $16^{\mathrm{e}}$ suppl. à Gallia, p. 83-84. 192.

31 Pi.ine, NH, III, 46; C. Nicolet, op. cil., p. 184-

32 Il remonte vraisemblablement, à notre avis, à l'époque césarienne : M. Christol., Chr. Goudinfau, op. cil., p. 90. Pour une appréciation comparable de l'œuvre césarienne supra note 17. L'un de nous avait déjà esquissé ce schéma : M. Christol, Le droit latin en Narbonnaise : l'apport de l'épigraphie (en particulier, celle de la cité de Nimesi, in : Epigrafia juridica romana. Actas del coloquio internacional AIEGL (Pamplona, 9-11 abril de 1987). Pampelune, 1989, p. 66-67 (= Les inscriptions latines de Gaule . Varbonnaise, Actes de la table-ronde de Nimes, 25-26 mai 1987, École antique de Nimes, n.s., 20, 1989. p. 87-89 avec plus de détails). derrière bien des oppida latina se cachaient des colonies latines ${ }^{33}$ ? Et que, surtout, la liste était valable pour le début de l'époque flavienne?

Mais l'intérêt de ce texte d'Arles est de donner plus de crédit à l'inscription d'Apt puisque les hypothèses qu'un auteur tel que A. L. F. Rivet pouvait déduire du titre de la colonie sont confirmées : la réalité de la phase julienne, entendue au sens large (triumvirale et augustéenne) est incontestable. Nous avons désormais, par l'épigraphie, la preuve qu'une phase de l'évolution institutionnelle de la cité d'Avignon s'étend entre le moment où celle-ci, anciennement "ville de Marseille" ${ }^{34}$, reçut le statut de colonie latine, entre 27 avant J.-C. et 14 après J.-C., et le moment où Hadrien couronna cette histoire par la création d'une colonie de droit romain.

Le lerminus post quem repose sur la mention Iulia Augusta dans la titulature de la cité. Ce constat rapproche l'histoire d'Avignon, telle qu'elle est à présent connue, de l'histoire de Riez et peut-être aussi de celle d'Aix-en-Provence. En revanche cette précision l'oppose à Apt, Carpentras et Carcassonne qui, pour l'instant, dans la documentation, ne sont que colonies Iuliae. En effet, si l'on peut estimer que dans certains cas, comme il en est à présent pour Avignon, une nouvelle inscription pourrait modifier la perspective d'étude, il n'en demeure pas moins qu'une enquête sur la titulature épigraphique des colonies de Narbonnaise apporte quelques enseignements significatifs; elle montre en particulier une forte régularité dans les énoncés.

\section{Apt (Pline : Apta Iulia Vulgentium)}

CIL XII, 1005 (Arles) : IIIIIIviro col. Iul. Aptae

CIL XII, 1114 (Apt) : IIIIvir., flam., augur. col. I. Apt.

CIL XII, $1116(\mathrm{Apt})$ : flamini, IIIIviro col. Iul. Apt. CIL XII, 1118 (Apt) : flaminic. Aug. col. Iulia Apta CIL XII, 1120 (Apt) : IIIIvir. C. I. Apt. (bis) et flam. Sans avoir à considérer les formes contractées ou raccourcies de cette titulature civique, il existe suffisamment de concordances dans la série rassemblée ci-dessus pour conclure que la fondation de la colonie latine est antérieure à 27 avant. .J.-C. ${ }^{35}$.

33 En ce sens Pali.u de Lessert, op. cit., p. 286. Du mème avis, A. Chastagnol, A propos du droit latin provincial, Iura, 38,1977 , p. 6.

34 Chr. Goudinfau, Sur un mot de Cicéron ou Avignon et le domaine de Marseille, in : L'Italie préromaine et la Rome républicaine, Mélanges offerts à Jacques Heurgon, Rome, 1976, I, p. 325-332.

35 Mais il n'est pas nécessaire d'intégrer la fondation d’Apt dans le plan césarien qu'évoque A. L. F. Rivfr, op. cit., 
2 Carpentras (Pline : Carpentorate Meminorum) CIL XII, 1239 (Orange) : [---] col. Iul. Mem.

En dépit de la minceur du seul témoignage connu pour l'instant, il n'y a aucune raison pour ne pas placer la fondation de la colonie durant la mème période.

\section{Carcassonne (Pline : Carcaso Volcarum)}

CIL XII. 5371 (Rieux-Mérinville, entre Narbonne et Carcassonne) : prail. C.I.C.

Pour cette cité, on suivra le raisonnement développé plus haut ${ }^{36}$.

\section{Avignon (Pline : Avennio Cavarum)}

Inscription inédite (Arles) : IIIIIIvir. Aug. col. Iul. Aug. Avennion.

Ici le titre de Iulia Augusta impose de placer la création de la colonie latine après 27 avant J.-C. (voir supra).

\section{Riez (Pline : Alabaece Reiorum Apollinarium) \\ CIL XII, 358 (Riez) : IIIIIIvir Aug. C.I.A.A. \\ CIL XII, 367 (Riez) : IIIIvir C.I.A.A. \\ CIL XII, 3991 (Nimes) : \\ decurion. col. Iul. Aug. Apollinar. Reior. \\ CIL XII, 408: (Nimes) :

$$
\text { |---/ col. Iul. August. Apollin. }
$$

Comme pour $\mathrm{Apt}$, il est inutile de prendre en considération les formes contractées ou les variantes. Les exemples sont suffisamment concordants : la fondation de la colonie latine se produisit à une date postérieure à 27 avant J.-C.. ${ }^{37}$.

\section{Aix (Pline: Aquae Sextiae Salluviorum)}

CIL XII, 705 (Arles) : IIIIIlvir Aug. col. Iul. Aquis CIL XII, 982 (Arles) : IIIIItvir. Aug. col. Iulia Aug. Aquis Sextis

CIL XII, 4414 (Narbonne): VIvir A/ug.J C.I.P. C....M. ell] Aq[uis] Sext/is]

p. 75 et p. $25 \overline{7}$ : une colonia lulia peut avoir été créce à partir du moment ou César le Jeune contròla la Transalpine, et jusquien $? 7$ a arant .J.-(:.

36 М. (iayrails. Varbonne antique des origines a la fin du $\mathrm{II}^{\circ}$ siècle. Paris, 1980), 8 "suppl. à la Revue archéologique de Varbonnaise, p. 322.

37 Du mème avis est A. I. F. Rivet (op. cit., p. 243). Pour sa part. (i. Barruol (Les Peuples préromains.... p. 218), envisage quayant reçu le droit latin de césar la cité serait devenue colonie romaine sous Auguste (cf. supra, pour ce schema, note :20). Renvovons aussi à l'introduction historique precédant lédition des inscriptions de Rie\% par les soins de I. A. Chastagnol (qu'il soit permis de le remercier pour nous avoir autorisé a consulter son ouvrage: Inscriptions latines de Varbonnaise, Antibes, Riez, Digne. 44r suppl. à Gallia, 1. 2. Paris, ed. C.NRs, 1992, 302 p.. fig.. 3 (artes).
CIL XII, 4124 (Narbonne) : IIIIIIvir. Aug. C.I.P. C.N.M. et Aquis Sextis

(.IL XI1. 4528 add (Narbonne): [sevir] Aug. C. $<I .>$ A. Aquis

Il s'agit, entre tous, du cas le plus délicat, car le plus grand nombre d'exemples sûrs ne comporte pas le cognomen Augusta. Aussi pourrait-on hésiter entre la première période et la seconde. Toutefois, si l'on etait tenté d'écarter le dernier texte provenant de Narbonne, il ne conviendrait pas de le faire à la légère, car, en dépit des conditions de sa transmission, il reste explicitement attaché à Aix-enProvence. 0 . Hirschfeld a proposé une restitution que nous retiendrons : elle consiste en la transformation du $\mathrm{F}$ de la copie en $\mathrm{I}^{38}$, ce qui fournit la titulature C. I. A. Aquis. De plus, si l'on voulait rejeter cette inscription sous prétexte qu'il faut procéder à une correction pour en établir le texte, que faire de la lettre qui sépare le C du A? Ainsi l'on n'est point obligé de privilégier la période antérieure à 27 avant J.-C. ${ }^{39}$. On ne peut non plus supposer que se serait déroulée une quelconque évolution à l'époque triumvirale puis à l'époque augustéenne : ne serait-elle pas contredite par le fait que l'autre inscription de Narbonne. postérieure à l'époque claudienne, n'attribue pas le surnom Augusta à la titulature de la cité d'Aix? si l'on observe que pour cette cité la documentation épigraphique provient de cités étrangères, on pourra estimer que les formes données à la titulalure revètent une diversité bien explicable. Il faut retenir celle qui est la plus complète, c'est-à-dire celle qui rappelle le rôle d'Auguste comme fondateur. Aix-en-Provence doit être une cité Iulia Augusta, comme Riez et Avignon ${ }^{40}$.

On examinera à part le cas de Ruscino, cité dotée aussi du droit latin, au moins jusqu'à l'époque de Vespasien ${ }^{41}$. Pomponius Mela, qui est antérieur à Pline l'Ancien ${ }^{42}$, lui donne le titre de colonia, mais on

\footnotetext{
3X Iddendum à CII XII, 1528, p. 8.77. Voir aussi, ibid., p. 930 .

39 On ne retiendra pas l'avis de A. L. F. Rivet (op. cil. p. $: 21: 2)$

10 Reprenant le dossier des sources litteraires, I). Roman (Aix-en-Provence et les débuts de la colonisation de droit romain en Ciaule du sud, Renue archéologique de Varbonnaise, 20,1987 , p. 185-190) défend l'hypothèse de la fondation d'une colonie latine au lendemain de la victoire sur les Salyens. Il est possible que les remaniements de l'époque triumvirale et de l'époque augustéenne aient tenu compte des situations antérieures, ou que parfois aussi ils les aient enfoui derrière le titre de fondation. Mais que faire de Pline qui. sous les Flaviens, maintient Aix-en-Provence parmi les oppida latina?

11 Pl.se, VH, III, 32 : Ruscino Latinorum.

42 Ponponus Mira, le Chorographia, 2, 5, 84; P.-.I. I)(val. La Gaule .... I. p. 344-346.
} 
se gardera de trop presser ce témoignage, comme dailleurs celui de Ptolémée. Ce dernier, en effet, lui attribue seulement la qualité générique de llónss, sans autre précision ${ }^{43}$. Il existe aussi un dossier épigraphique. mais il est d'interprétation délicate. En effet, en publiant un fragment réduit à trois lettres (C.I.R. selon sa lecture), P. Thiers avait estimé qu il livrait le titre de la colonie (c(olonia) I(ulia) $R($ uscino $))^{44}$. On pourrait en déduire que Ruscino fut d'abord une colonie latine ${ }^{45}$, fondée antérieurement à 27 avant J.-C. (colonie Iulia). Mais la lecture de la dernière lettre n'est pas assurée, ni la signification du fragment évidente, car ce qui demeure du texte initial représente trop peu de chose ${ }^{46}$. Mème si. récemment, M. Gayraud a préféré l'écarter du dossier ${ }^{47}$, nous le conserverons, mais en reconnaissant que si l'inscription peut se référer à la dénomination d'une cité, le développement c(olonia) I(ulia) R(uscino) ne simpose pas absolument, car la troisième lettre n'est pas forcément la lettre $R$ (on pourrait penser à $\mathrm{F}$ ou B). Mais il existe aussi une seconde inscription, publiée par $P$. Thiers avec la précédente, et reprise après lui par E. Espérandieu et par $\mathbf{M}$. Gayraud. Elle fait allusion à un magistrat municipal, bénéficiaire d'un hommage en lieu public, peut-être élevé par ses héritiers après la permission de l'ordo ${ }^{48}$. Toutefois la restitution et son interprétation sont, de mème, délicates. P. Thiers supposait que le personnage avait été duumvir à Ruscino puis à Narbonne. V. Gayraud, qui apportait une édition du texte plus satisfaisante, estimait pour sa part que le notable

4:3 Pтolt́víte, Géographie, 2, 10, 6. Sur le témoignage de cet auteur et sur celui de Pomponius Mela, M. Gayratid, les inscriptions de Ruscino, in: G. Barrcol el alii. Ruscino. Chateau-Roussillon, Perpignan (Pyrénées-Orientales). I. État des travaur el recherches en 1957. Paris, 1980, $7^{\circ}$ suppl. a la Revue Archéologique de Narbonnaise. p. 96.

14 P. Thiers, Bulletin archéologique du Comité des Tranaux Historiques, 1913, p. 1933 (d'où ILGN 637).

15 Le seul élément sùr qui rapproche Ruscino des autres cités de droit latin en Varbonnaise est linscription des notables dans la tribu Voltinia. A. L. F. River, op. cit., p. 136. ne doute pas un instant que Ruscino lùt colonie latine "in Augustan times".

46 On se référera désormais à M. GaYraud, Les inscriptions..., n" 28, p. 92.

47 V. (iayna('D), Les inscriptions ..., p. 92 et p. 94.

18 P. ThIERs, op. cil., 1913. p. 219 (IIG.V 634) = 11. (iayraci). Les inscriptions.... $\mathrm{n}^{\circ} 26$, p. 91 (avec photo). Cous lisons : /-P/om/p/ei/o---///---/vir. I/v/---///--flJamin. bis /--l / /--PJom/p/ei/us/ Lep/---/ / /--he/red/es---/. On pourrait done lire a la dernière ligne conservée non /impensam/ red/emil/ mais /he/red/es/. Provenant de lintérieur de la ville. on considèrera qu il s'agit dun hommage de caractère public. donc élevé avec la permission des décurions (l.d.d.d.). aurait été quattuorvir à Ruscino, puisqu'il s'agit d'une cité de droit latin, et par la suite duumvir à Narbonne. Aussi restituait-il : [---IIIIJvir(o), IIv(iro) [C.I.C.P.Y.M.?]. Nais on pourrait renoncer à lire sur l'inscription un cursus qui se serait déroulé dans deux cités différentes, mème voisines. Toute la carrière ne serait-elle pas locale, surtout si l'on admet que, puisqu'elle provient d'un espace public, l'inscription montrerait la vigueur de la vie politique de la cité. Ne pourrait-on donc proposer de restituer de la sorte: / praef(ecto) pro (duo)]vir(o), (duo)v[ir(o) ---J, ou bien : [(duo)]vir(o), (duo)v [ir(o) quinquennali---]? Ce serait alors le signe d'un changement de statut de Ruscino, plus précisément celui d'une élévation au rang de colonie de droit romain. Et dans ce cas l'on se trouverait devant une évolution comparable à celle d'Avignon, sans que l'on dispose cependant de la possibilité d'être plus précis en matière chronologique : seul le texte de Pline devrait vraisemblablement faire rejeter l'élévation de la cité après le règne de Vespasien ${ }^{49}$. Mais de toute façon, cette reconstruction de l'histoire de Ruscino demeure très aléatoire ${ }^{50}$.

En somme, le développement des colonies latines de Narbonnaise aurait pu suivre le même schéma progressif que la mise en place des colonies de droit romain. Pour celles-ci on relève une succession d'implantations qui s'étendent de l'époque césarienne, aver Narbonne et Arles ${ }^{51}$, jusqu'aux années précédant immédiatement 27 avant J.-C., avec Fréjus ${ }^{52}$. Pour les colonies latines, à la phase césarienne, dans laquelle, grâce à la documentation numismatique et aux textes, on peut placer au moins Nimes et Vienne ${ }^{53}$, succéderaient deux autres phases,

49 M. Gayraud (Les inscriptions.... p. 94) place l'inscription de ce notable municipal après le règne de Vespasien, puisqu elle mentionnerait le flaminat provincial.

50 M. Gayraud (Les inscriptions .... p. 96-97) s'était engagé dans cette voie avant de se rallier à l'idée d'une absorption de Ruscino dans la colonie de Narbonne, où aurait été exerce le duumvirat mentionné dans l'inscription. Cf. aussi M. (iayrat. . Narbonne .... p. 323-324 et p. 400. Sur ce point. A. L. F. Rivet (op. cit.. p. I(1) et p. 136) demeure hésitant.

5l C'est ainsi que l'on peut interpréter Suétone (Tib., 4): - Chr. Goedinead, op. cil., Gallia, 44, 1986, p. 171-173.

52 J. Gascove Quand la colonie de Fréjus fut-elle fondée?. Latomus, 41, 1982, p. 132-145. Béziers et Orange, colonies romaines, créées vraisemblablement en 36 et 35 avant J.-(. (A. Piganiol.. Les documents cadastraux .... p. 79-84), sont des colonies Iuliae.

53 Chr. Gocminfat, op. cil., Gallia, 44, 1986, p. 172 ; Ib.. Les textes antiques sur la fondation et la topographie de Lugdunum, in : Chr. Goldonfal: (dir.), Aux origines de Lyon, Lỵon. 1989, Documents d'Archéologie en Rhòne-Alpes, 2. p. $2(6 ;-$ M. Cinristol, Chr. Gouniveav, op. cil., p. 92. 
d'après l'apport de la documentation épigraphique : l'une durant la période triumvirale (les colonies Iuliae), l'autre durant la période augustéenne (les colonies Iuliae Augustae) $)^{54}$. A la première appartiendraient Apt, Carpentras et Carcassonne, peut-être Ruscino; à la seconde se rattacheraient Avignon, Riez et Aix-en-Provence ${ }^{55}$. Pour sa part, la numismatique apporte des compléments qui semblent bien

54 Les sources littéraires (Dion Cassius) et épigraphiques (Res Gestae) font allusion a plusieurs vagues de colonisation qui se prolongent jusqu'aux années 16-14 avant $\mathrm{J}$.-C. : M. Christol, Chr. Goudinfau, op. cit., p. 101. Chacune d'entre elles aurait apporté sa part de colonies latines en Narbonnaise.

55 Le cas d'Alba des Helviens n'est pas encore tranché, bien que d'après les institutions municipales faisant connaître le quattuorvirat, il s'agisse d'une colonie latine : voir en dernier J. Gascou, Duumvirat, quattuorvirat et statut dans les cités de Gaule Narbonnaise, in : Epigrafia. Actes du colloque en mémoire de Altilio Degrassi, Rome, 1991, p. 547-563, surtout p. 560 . s'intégrer à l'évolution générale que nous venons d'évoquer. De toute façon, on peut espérer ajouter tòt ou tard aux listes actuelles de nouvelles cités, car il existe encore de nombreuses incertitudes sur l'èvolution du statut de plusieurs autres communautés de Narbonnaise.

Michel Christol et Marc HeiJmans

I. B. - Les deux documents mentionnés à la note 3 sont désormais publiés: M. Christol, M. Heismans, Nouvelles inscriptions d'Arles, Documents d'Archéologie Méridionale, 14, 1991, p. 355-361. J. Gascou, pour sa part, a défendu l'authenticité de CII XII, 1120 (cf. note 15) : Le statut d'Avignon d'après un prétendu faux épigraphique de la cité d'Apt (Vaucluse), Revue archéologique de Narbonnaise, 23, 1990, p. $225-233$. 